\title{
Calibration-free quantitative elemental analysis of meteor plasma using reference laser-induced breakdown spectroscopy of meteorite samples
}

\author{
Martin Ferus $^{1}$, Jakub Koukal ${ }^{2}$, Libor Lenža ${ }^{1,2}$, Jiří Srba $^{2}$, Petr Kubelík ${ }^{1,3}$, Vojtěch Laitl ${ }^{1}$, \\ Ekaterina M. Zanozina ${ }^{1,3}$, Pavel Váňa ${ }^{1}$, Tereza Kaiserová ${ }^{1}$, Antonín Knížek ${ }^{1,4}$, Paul Rimmer ${ }^{5,6}$, \\ Elias Chatzitheodoridis ${ }^{7}$, and Svatopluk Civiš ${ }^{1}$
}

\footnotetext{
${ }^{1}$ J. Heyrovský Institute of Physical Chemistry, Academy of Sciences of the Czech Republic, Dolejškova 3, 18223 Prague 8, Czech Republic

e-mail: svatopluk.civis@jh-inst.cas.cz

2 Observatory Valašské Meziř́ičí, Vsetínská 78, 75701 Valašské Meziříčí, Czech Republic

${ }^{3}$ Department of Radiation and Chemical Physics, Institute of Physics, Academy of Sciences of the Czech Republic, Na Slovance 1999/2, 18221 Prague 8, Czech Republic

${ }^{4}$ Charles University in Prague, Faculty of Science, Department of Physical and Macromolecular Chemistry, Albertov 2030, 12840 Prague 2, Czech Republic

${ }^{5}$ Battcock Centre for Experimental Astrophysics Astrophysics Group Cavendish Laboratory, J.J. Thomson Avenue, Cambridge CB3 OHE, UK

${ }^{6}$ Biomedical Research Council Laboratory of Molecular Biology, Francis Crick Ave, Cambridge CB2 OQH, UK

7 National Technical University of Athens, School of Mining and Metallurgical Engineering, 9 Heroon Polytechneiou str., 15780 , Zografou, Athens, Greece
}

Received 24 October 2016 / Accepted 3 December 2017

\begin{abstract}
Aims. We aim to analyse real-time Perseid and Leonid meteor spectra using a novel calibration-free (CF) method, which is usually applied in the laboratory for laser-induced breakdown spectroscopic (LIBS) chemical analysis.

Methods. Reference laser ablation spectra of specimens of chondritic meteorites were measured in situ simultaneously with a highresolution laboratory echelle spectrograph and a spectral camera for meteor observation. Laboratory data were subsequently evaluated via the $\mathrm{CF}$ method and compared with real meteor emission spectra. Additionally, spectral features related to airglow plasma were compared with the spectra of laser-induced breakdown and electric discharge in the air.

Results. We show that this method can be applied in the evaluation of meteor spectral data observed in real time. Specifically, CF analysis can be used to determine the chemical composition of meteor plasma, which, in the case of the Perseid and Leonid meteors analysed in this study, corresponds to that of the C-group of chondrites.
\end{abstract}

Key words. astrochemistry - techniques: spectroscopic - meteorites, meteors, meteoroids - plasmas

\section{Introduction}

In-depth analysis of meteor spectra can be used for basic qualitative and quantitative elemental analyses and characterizations of meteoroids and their parent bodies. Instead of detailed descriptions of real quantitative chemical compositions, most of the early and current studies have been focused on the identification and comparison of intensities of the dominant emission lines, such as $\mathrm{Fe}, \mathrm{Cr}, \mathrm{Ca}, \mathrm{Mg}$, and $\mathrm{Na}$, or other elements such as $\mathrm{H}, \mathrm{Li}, \mathrm{Al}, \mathrm{Si}, \mathrm{Ca}, \mathrm{Ti}, \mathrm{Cr}, \mathrm{Mn}, \mathrm{Co}, \mathrm{Ni}$, or $\mathrm{Sr}$ (Abe et al. 2005; Madiedo et al. 2014a; Vojacek et al. 2015). Exact elemental quantitative analyses are only occasionally published Borovička et al. (1999); Jenniskens (2007); Madiedo et al. (2013). Several observations Berezhnoy \& Borovicka (2010) of very bright bolides using highly sensitive instruments also provided not only the emission spectra of atoms but also of radicals, molecules, and molecular ions (e.g. $\mathrm{N}_{2}^{+}, \mathrm{CN}, \mathrm{C}_{2}, \mathrm{OH}, \mathrm{SiO}$, etc.) and, more recently, such binary compounds as $\mathrm{FeO}, \mathrm{CaO}, \mathrm{AlO}$, and $\mathrm{MgO}$ (Borovička \& Berezhnoy 2016). Usually, quantitative analysis of elemental abundances in a meteor plasma is achieved via comparison of computed spectra with observed spectra (Borovička \& Betlem 1997). This procedure is very complicated and requires large databases of spectral features of all the elements and a deep understanding of the optical behaviour of the meteor plasma. However, public spectral databases such as NIST (Kramida et al. 2015) or The Atomic Linelist (2016) are presently incomplete. Particularly, in accordance with our experiences, data regarding Einstein emission coefficients are presently lacking for many important spectral lines of various elements. As shown below, this problem is not the only important issue connected with the spectroscopic investigations of the elemental composition of plasmas.

In the current study, we introduce a different procedure for determining the elemental abundances in a meteor plasma. First, in our laboratory, we employed comparative analysis using the laser ablation of real samples of meteorites (LIBS) to simulate a meteor fireball under laboratory conditions. Additionally, the airglow emission is simulated using electric discharges and 
laser-induced breakdown in air. For further analysis of elemental abundances in Perseid and Leonid spectra, as well as in the plasma induced on the surfaces of meteorite specimens using a laser, CF data evaluation procedure, which is commonly used in metallurgy, was carried out.

The main advantage of comparative measurements using LIBS lies in the applicability of this method for the real-time in situ analysis of any sample of a real meteorite without any preceding treatment, preparation or isolation (Hornackova et al. 2014). Plasma is generated in the laboratory under strictly defined conditions, and atomic or molecular species are evaporated from a relatively large volume in a manner similar to that induced by plasma generated during meteoroid descent. Therefore, only for the purposes of qualitative analysis, the spectra can be easily and directly compared to the spectra of meteors without any need for computing artificial spectra with the use of large databases. This makes LIBS suitable for qualitative analyses of meteor composition.

However, the situation is different if quantitative elemental determination is also required. In fact, from the spectroscopic point of view, real meteor spectroscopy and laboratory metallurgical analysis face several complications. In a series of previous studies dealing with the LIBS method, it was demonstrated that the emission intensity of a particular spectral line of a studied analyte depends not only on the physical parameters of the spectral transition and the quantity of the emitting element, but also on the matrix in which the element is embedded Tognoni et al. (2010). This matrix influence leads to the need for calibration curves or matrix-matched standards, which, in some practical situations, including for samples of meteorites, are simply unavailable. In Ciucci et al. (1999) the authors proposed a novel procedure called CF laser-induced breakdown spectroscopy (CF-LIBS) to overcome the mentioned matrix effect. Instead of looking at the matrix as an independent problem, the CF-LIBS method is based on the direct analysis of emission lines of a particular analyte embedded in the matrix.

In the current study, we have applied the CF method to interpret the spectra of two bright bolides - Perseid and Leonid. These meteors have been analysed independently in previous works using model spectra. Therefore, their chemical compositions are known and can be easily compared with the results produced by $\mathrm{CF}$ calculations. Moreover, as mentioned above, all the data are compared with reference experimental spectra obtained from meteorite ablation, laser-induced breakdown (LIDB) in the air and glow discharge in air.

\section{Experimental procedures}

\subsection{Ablation of solid samples}

Meteor plasma was simulated in our laboratory via the laser ablation of meteorite samples using a Lambda Physik (ArF) excimer laser. The laser emits 10 -ns pulses with a wavelength of $193 \mathrm{~nm}$ and energy of $200 \mathrm{~mJ}$. The laser beam was focused using a calcium fluoride lens (focal length of $50 \mathrm{~mm}$ ) onto a solid target (sample of meteorite) attached to an $X Y Z$ rotation stage. The system was placed in a vacuum interaction chamber equipped with a collimator connected directly to a highresolution echelle spectrograph (ESA 4000, LLA Instruments $\mathrm{GmbH}$, Germany). The chamber was cleaned with nitrogen inert gas and evacuated to pressure of 2 Torr. The low resolution spectrum was simultaneously measured using the meteor spectral camera.

\subsection{High-resolution echelle spectrograph}

The system of the ESA 4000 echelle spectrograph (LLA Instruments $\mathrm{GmbH}$, Germany) is a highly sensitive optoelectronic device, which is extremely susceptible to mechanical and optical stress, particularly with regard to an ICCD-camera. The spectrograph allows for the simultaneous measurement of complex spectra within the entire 200-780 nm UV/VIS - region, with an effective resolution ranging from $0.005 \mathrm{~nm}(200 \mathrm{~nm})$ to $0.019 \mathrm{~nm}$ $(780 \mathrm{~nm})$. In our measurement, the spectrograph was triggered with an 800-ns laser pulse and to start the measurement with a delay of $4 \mu \mathrm{s}$, with a gate open for $5 \mu \mathrm{s}$ with an accumulation of 3500 counts and 200 accumulations of the signal.

\subsection{Meteor spectral camera}

The optical set-up of the camera for meteor spectrographic analysis was based on direct light dispersion by a holographic grating, and the emission spectrum was imprinted in the original photography of the emitting object (a laser or discharge plasma in the laboratory, or the meteor during observation). The current meteor spectral cameras are based on QHY5LII-M hardware, with a $1280 \times 960$ px CMOS chip and an objective Tamron (f/1,00; F/3-8 mm) equipped with a 1000 lines/mm grating, which allows a resolution of up to $0.97 \mathrm{~nm} \mathrm{px}^{-1}$.

\subsection{Comparative experiments with laser induced breakdown and discharges in air}

Comparative experiments with atmospheric gases have been performed using Laser Induced Breakdown in air and electric discharges. The emission spectra of electric discharge plasma from a positive column discharge in the air were simultaneously observed using the echelle spectrograph and the meteor spectral camera. The positive column discharge tube $(25 \mathrm{~cm}$ long with an inner diameter of $12 \mathrm{~mm}$ ) was covered with a glass outer jacket and equipped with $\mathrm{CaF}_{2}$ windows that are transparent within the operation range of both spectrographs. The pulsed discharge was maintained by a high-voltage transistor switch (HTS 81, Behlke electronic GmbH, Frankfurt, Germany) applied between the stainless steel anode and the grounded cathode. The air plasma was cooled by water in the outer jacket of the cell. The voltage drop across the discharge was $1200 \mathrm{~V}$, and the current was $250 \mathrm{~mA}$.

The spectra of LIDB in the air were recorded using both spectrographs by focusing the laser radiation in the air. Laser pulses of $20 \mathrm{~Hz}, 450 \mathrm{~mJ}$ and $1064 \mathrm{~nm}$ generated by a Quantel Q-smart $450 \mathrm{Nd}$ :YAG laser were focused by a $10 \mathrm{~cm}$ coated quartz lens.

\section{Calculations}

The procedure of CF analysis applied in this study for the calculation of the composition of three samples of meteorites and the interpretation of meteor spectra is described in detail in previously published literature (Ciucci et al. 1999; Tognoni et al. 2010; Hornackova et al. 2014; Takahashi et al. 2015; Ozdin et al. 2015; Dell'Aglio et al. 2014; De Giacomo 2011). The data processing can be summarized as follows: under specific conditions in a plasma, local thermodynamic equilibrium (LTE) is achieved in the temporal and spatial observation window and the plasma is assumed to be representative of the composition for the unperturbed target composition. In this case, then the plasma 
can be assumed to be a spatially homogeneous source of radiation. Spectral lines included in the calculation are optically thin, and, if measured, the spectral range involves detectable, isolated and representative lines from all elements present in the target, excited levels are populated according to the Boltzmann distribution, and the ionization states satisfy the Saha-Boltzmann equilibrium equation. In such an approach, the atomic composition can be determined via the $\mathrm{CF}$ method based on the following expression for the integrated intensity of the emission line $I(v)$ in SI units:

$I(v)=\frac{F C_{S} A_{i j} g_{i} v_{i j} h}{4 \pi Q_{S}(T)} \exp \left(\frac{-E_{i}}{k_{\mathrm{B}} T}\right)$,

where $F$ is an experimental parameter that accounts for the optical collection efficiency and plasma density, $C_{S}$ represents the concentration of the emitting neutral atom $\mathrm{S} \mathrm{I}, A_{i j} g_{i}$ is a product of the Einstein emission coefficient and the degeneracy of the transition between the $i$ and $j$ state, $v_{i j}$ is the frequency of the transition, $h$ is the Planck constant, $E_{i}$ is the upper level energy, $k_{\mathrm{B}}$ is the Boltzmann constant, $T$ is the plasma temperature, and $Q_{S}$ is the appropriate partition function, taken in this study from the Atomic Spectra Database of the National Institute for Standards and Technology (Kramida et al. 2015). This function, for every particular element $S$ at temperature $T$, is equal to the sum of the upper level degeneration $g_{i}$ factors as

$Q_{S}^{0}(T)=\sum_{i} g_{i} \exp \left(\frac{-E_{i}}{k_{\mathrm{B}} T}\right)$.

In this expression, $g_{i}=2 J_{i}+1$ denotes the electronic degeneracy factors, with $J_{i}$ being the total angular (orbital + spin) momentum quantum number of $E_{i}$. All the values can be found in the tables (Kramida et al. 2015).

We should note that, for elemental analysis, a mixing ratio is commonly used to express the abundance of a particular element. In this study, we scaled the elemental abundance to magnesium, such that the mixing ratio of element is expressed as $A: x(A)=n(A) / n(\mathrm{Mg})$.

Equation (1) can be reformulated as a linear equation in a two-dimensional space called the Boltzmann plane:

$\ln \left(\frac{I(v)}{A_{i j} g_{i} v_{i j}}\right)=\frac{-1}{k_{\mathrm{B}} T} E_{j}+\ln \left(\frac{F C_{S}}{Q_{S}(T)} \frac{h}{4 \pi}\right)$.

In fact, this linear expression represents the Boltzmann plot, which can be directly used to estimate the plasma temperature for neutral atomic species. The electron temperature can be determined using the Saha-Boltzmann plot. From the slope $K$ of this fit, the plasma temperature $T$ can be determined as

$T=\frac{1}{k_{\mathrm{B}} K}$.

For ionized species S II, the expression is modified to

$$
\begin{aligned}
\ln \left(\frac{I(v)}{A_{i j} g_{i} v_{i j}}\right)-\ln \left(\frac{2\left(2 \pi m_{\mathrm{e}} k_{\mathrm{B}} T\right)^{\frac{3}{2}}}{N_{\mathrm{e}} h^{3}}\right)= & \frac{-1}{k_{\mathrm{B}} T_{\mathrm{e}}}\left(E_{j}+E_{\mathrm{ion}}\right) \\
& +\ln \left(\frac{F C_{S}}{Q_{S}(T)} \frac{h}{4 \pi}\right),
\end{aligned}
$$

where $N_{\mathrm{e}}$ is the electron density, $T_{\mathrm{e}}$ is the electron temperature, and $E_{j}+E_{\text {ion }}$ is a sum of the upper-level energy of the ionized species and its ionization energy $E_{\text {ion }}$. Again, from the linear fit, the electron temperature can be estimated in the same manner as Eq. (4):

$T_{\mathrm{e}}=\frac{1}{k_{\mathrm{B}} K}$.

Here, we note that similar equations can be derived for all species contained in the plasma. Under the conditions of LTE plasma, all the species should exhibit the same or comparable excitation and electron temperature:

$T=T_{\mathrm{e}}$.

The biggest advantage of this analysis is that it involves plotting lines from different ionization stages of the same element into one graph and that it is possible to directly determine whether the LTE condition is achieved. In the non-LTE plasma, such a linear plot cannot be assembled.

In addition to Eq. (7), the condition of LTE is additionally expressed by the McWhirter criterion:

$N_{\mathrm{e}}>1.6 \times 10^{12} T^{\frac{1}{2}}\left(\Delta E_{n m}\right)^{3}$.

This defines the minimum electron density needed for LTE plasma. In this equation, $T$ is again the temperature, and $\Delta E$ is the highest energy gap between adjacent levels (here, in $\mathrm{eV}$ ). According to this expression, the minimal electron density of the laser-induced plasmas is of the order of $10^{16} \mathrm{~cm}^{-3}$.

The electron density of the plasma itself can be calculated using the Lorentzian fit of a particular emission line found at position $v_{0}$ and profiled by the Stark broadening mechanism and subsequent determination of its full width at half maximum (FWHM) $\gamma$ :

$\Phi(v)=I(v) \frac{\gamma / 4 \pi^{2}}{\left(v-v_{0}\right)^{2}+(\gamma / 4 \pi)^{2}}$

where $\Phi(v)$ represents the intensity as a function of frequency $v$ and the integral intensity of the line $I(v)$. Because spectral line broadening is mainly governed by collisions of the charged species, electron density may be calculated by using the relation

$N_{\mathrm{e}}=\frac{2 \Omega}{F W H M}$,

where $\Omega$ is the electron-impact parameter. If the ionic contribution is neglected, as well as its slow change with the electron temperature, this parameter can be taken for any particular line and the electron density can be estimated. Konjević (2002) reported on the experimental data for the Stark widths of spectral lines and the electron impact parameters $\Omega$ for several non-hydrogenic atoms.

If all the conditions of the LTE plasma are satisfied, we can insert the following substitutions into (3):

$$
\begin{aligned}
& Y=\ln \left(\frac{I(v)}{A_{i j} g_{i} v_{i j}}\right), \\
& m=\frac{-1}{k_{\mathrm{B}} T}, \\
& q_{S}=\ln \left(\frac{F C_{S}}{Q_{S}(T)} \frac{h}{4 \pi}\right) .
\end{aligned}
$$


Again, this series of equations can be written as a linear equation in a two-dimensional space called the Boltzmann plane:

$$
Y=m E_{j}+q_{S},
$$

where $q_{S}$ is related to the concentration $C_{S}$. Similar equations are then written for all the selected emission lines of species in the plasma. The plasma temperature is taken from the Boltzmann and Saha-Boltzmann fits according to Eqs. (3)-(6). Because $\frac{h}{4 \pi}$ represents only the ratio of physical constants, the concentration of all the species can be calculated using the intersection value $q_{S}$ of the linear regression in the Boltzmann plane:

$C_{S}=\frac{Q_{S}(T)}{F} \exp \left(q_{S}\right)$

The experimental factor $F$ representing optical collection efficiency must be calculated by normalizing the sum of all species concentrations:

$\sum_{S} C_{S}=\frac{1}{F} \sum_{S} Q_{S}(T) \exp \left(q_{S}\right)=1$.

In conclusion, the method of $\mathrm{CF}$ analysis summarized above represents a method, which separates the experimental factor $F$ from the data. In principle, it should be possible to calculate the mixing ratio of any particular element using only a pair of single lines. However, the matrix and other factors make it impossible to yield reliable results using such a simple method. Instead, the $\mathrm{CF}$ method directly incorporates the matrix dependence in the calculation algorithm by introducing the $F$ factor. Then, if we use a sufficient number of lines of two particular elements, their mixing ratio can be directly estimated. In this study, we used the mixing ratio relative to magnesium (as discussed above).

\section{Results and discussion}

\subsection{Comparative CF and spectroscopic analysis of chondrite samples}

In the first step of the comparative analysis, spectra of the ablation plasma of three meteorite samples, Dhofar 1764 (group CV3), Dhofar 1709 (group LL4) and Porangaba (group L4), were recorded using a high-resolution echelle spectrograph and a meteor spectral camera of the same type and technical parameters as those used for the observation of meteors. In Fig. 1, both the high- and low- resolution spectra are indicated.

Using the ESAWIN software (analysis of the data and operation using an echelle ESA high-resolution spectrograph; 2015), the NIST Atomic Spectra Database (Kramida et al. 2015) and The Atomic Linelist (The Atomic Linelist 2016), we assigned and compiled 1003 spectral lines with sufficient intensity. Within this line-list, 475 of the lines can be designated as prominent. The positions of the most important features are highlighted in Fig. 1. The prominent spectral lines of neutral atoms belong to the strong series of $\mathrm{Fe} I$ and $\mathrm{Cr}$ I lines, several strong lines of $\mathrm{Al} \mathrm{I}, \mathrm{Mg}$ I, Si I, and $\mathrm{Na} \mathrm{I}$, and weaker emissions of Mn I, Ti I, Ni I. Additionally, ions of Ca II and Mg II have been observed by Ozdin et al. (2015); Dell'Aglio et al. (2010). In fact, such qualitative analysis of the ablation spectra is in perfect agreement with that published by Borovička \& Betlem (1997) for the analysis of Perseid spectra. In their work, they also observed $\mathrm{Na}$ I, Fe I, Mg I, Cr I, Ca I, and Mn I series at similar wavelengths
(Borovička \& Betlem 1997). However, we did not observe such a strong Si II and Mg II emission in the ablation plasma.

Subsequently, the high-resolution data measured by the echelle spectrograph for all the specimens of chondritic meteorites mentioned above were processed using the $\mathrm{CF}$ method to obtain quantitative results intended for further comparison with the meteor spectra. In the first step, the electron density was calculated using Eq. (10). This parameter also shows whether the McWhirter criterion (8) for LTE plasma is satisfied. As mentioned above, the line profiles in the LIBS plasma are influenced mainly by Stark broadening caused by collisions and energy distribution driven by electrons. We assumed that the pressure broadening is not dominant. Comprehensive studies (Iida 1990; Effenberger \& Scott 2010) have shown that emission intensity varies primarily as a function of the electron density and plasma temperature. At vacuum conditions (about $10^{-5}$ Torr), the LIBS spectrum is usually less intense than the LIBS spectrum taken under atmospheric conditions. This behaviour is likely the result of the decreased electron density. On the other hand, at reduced pressures, plasma generated with a laser pulse is expanding within a less dense atmosphere, which results in a less dense shock wave. The reduced density in the shock wave results in reduced plasma shielding; allowing more photons to reach the sample, which increases the ablation efficiency. This behaviour indicates that the optimum pressure is yet to be determined. In our study, we have estimated optimal ablation pressure to be of 2 Torr, which is in agreement with previous assumptions (Iida 1990; Effenberger \& Scott 2010).

Figure 2 shows the Lorentzian fit of the Fe I 426.03 nm emission line for all the ablation spectra. For this clearly detectable line, the line parameter was found to be of $\Omega=0.11 \AA / \mathrm{nm}$ Konjević (2002). Using these values and the FWHM of this line in all three measurements, estimated using the fit, we obtained from Eq. (9) a mean electron density of $3.02 \times 10^{16} \mathrm{~cm}^{-3}$. Based on this result, we can state that the McWhirter criterion for the LTE plasma is satisfied.

Particular results for each meteorite are shown in Fig. 2. To demonstrate the significant contribution of the Stark broadening, we compare our results with the FWHM parameter given by the Doppler broadening using the formula (again, in SI units)

$F W H M=\frac{2 v_{0}}{c} \sqrt{\frac{2 k_{\mathrm{B}} T \ln 2}{m}}$,

where $v_{0}$ is the frequency of the transition, $c$ is the speed of light, $k_{\mathrm{B}}$ is Boltzmann constant, and $m$ is the atomic mass of the element (in the case of polyatomic species, reduced mass must be used instead). For the line mentioned above, the result is $6.14 \times 10^{9} \mathrm{~Hz}$, which corresponds to $0.0037 \mathrm{~nm}$. This result shows that Doppler broadening is in fact ten times smaller than the total FWHM corresponding to the Stark broadening given by collisions with electrons.

The excitation temperature from the experimental highresolution spectra was estimated using the both plots of Eq. (18) selected well-separated and prominent Fe I and Fe II lines in the spectra. Each individual line of Fe was fitted with a Lorentz profile, and the integral intensity was calculated. For the neutral lines, the values for the $X$ - and $Y$-axes are plotted according to Eq. (3), as follows:

$X: \ln \left(\frac{I(v)}{A_{i j} g_{i} v_{i j}}\right)$,

$Y: E_{j}$ 

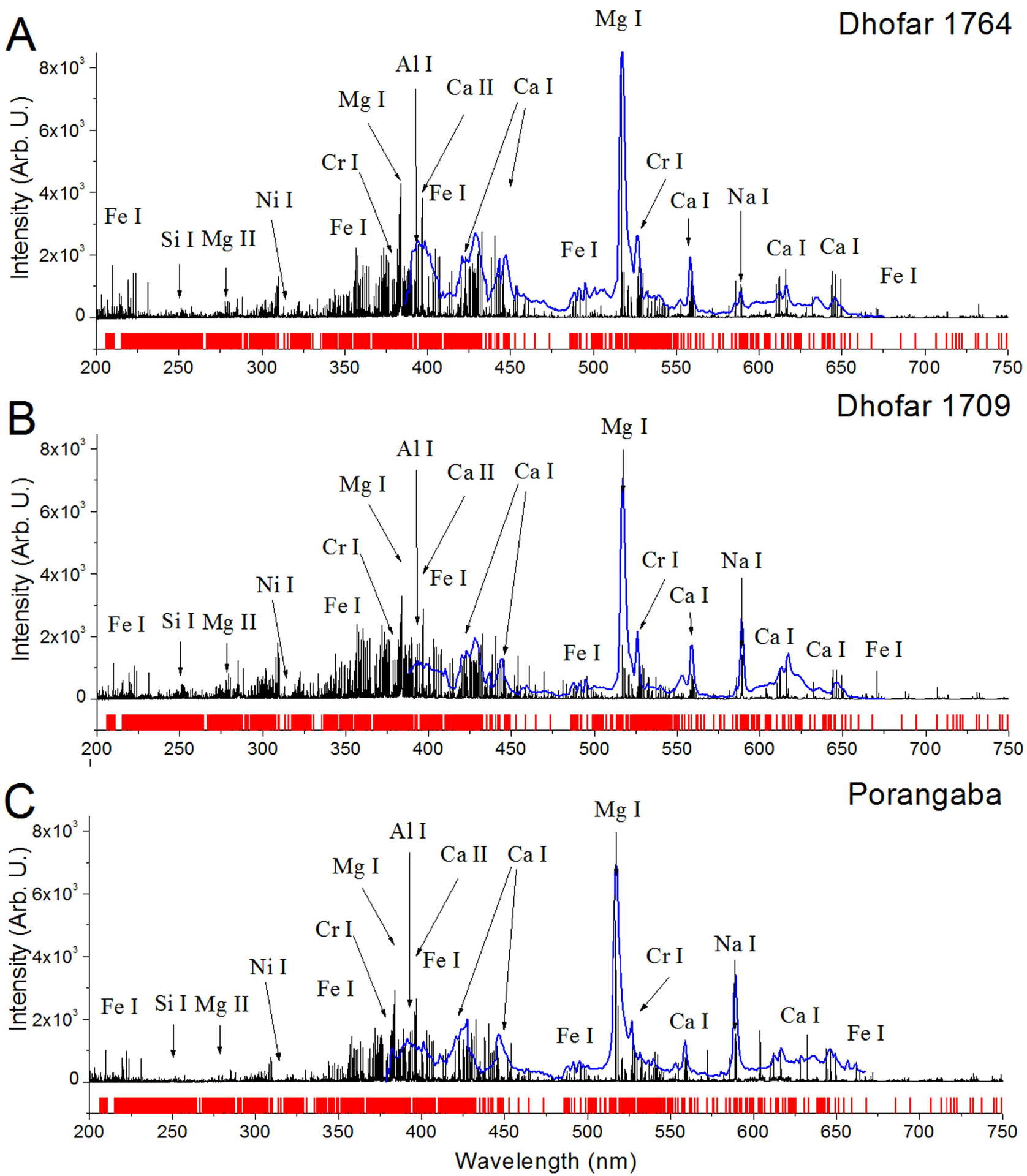

Fig. 1. Global survey of ablation emission spectra of all three samples of meteorites including the assignment of the most prominent lines. The spectra also contain many coinciding Fe lines. For better clarity, their positions are indicated separately by the red sticks below each graph. In the figure, the spectra recorded by the meteor spectral camera and the high-resolution echelle spectrograph are shown in blue and black respectively.

In the case of ionized Fe II lines (listed in the Supporting Information), the co-ordinates in the following graphs are depicted as follows:

$X:\left(E_{j}+E_{\text {ion }}\right)$,

$Y: \ln \left(\frac{I(v)}{A_{i j} g_{i} v_{i j}}\right)-\ln \left(\frac{2\left(2 \pi m_{\mathrm{e}} k_{\mathrm{B}} T\right)^{\frac{3}{2}}}{N_{\mathrm{e}} h^{3}}\right)$.
The slopes correspond to the excitation and free electron temperature $T_{\mathrm{e}}$, which is calculated using Eq. (6). The temperatures fitted for neutral $\mathrm{Fe}$ I lines within the all three samples correspond to the global fit in the Saha-Boltzmann plot for selected strong and well-defined neutral Fe I and ionized Fe II lines. The criterion of LTE (Eq. (7)) is also satisfied because the overall electron temperature corresponds to the temperature estimated using neutral atoms. As we can see in Fig. 3, all the data can be fitted linearly. 

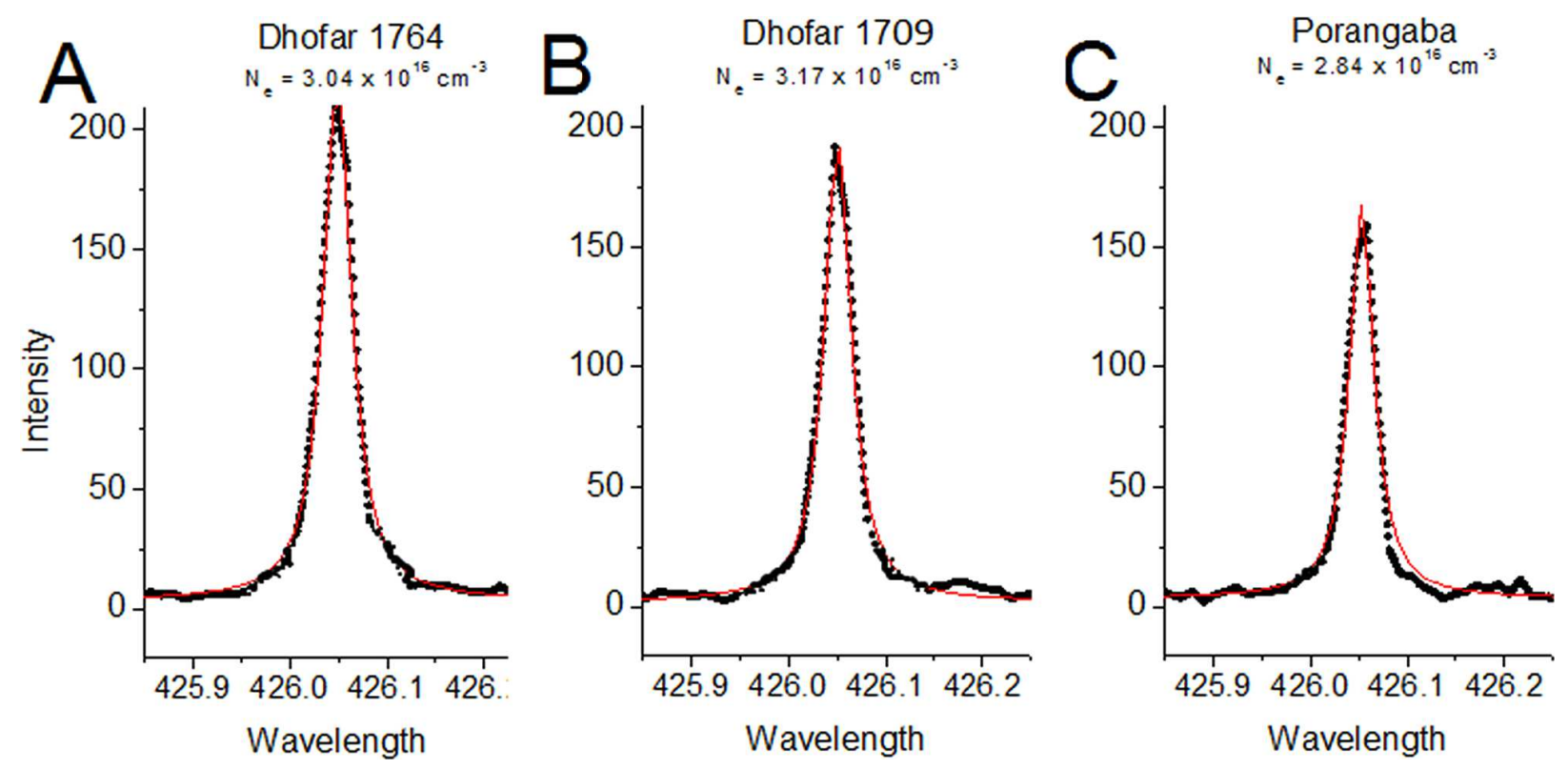

Fig. 2. Lorentzian fits of the Fe I emission line for all three ablation samples. The estimated mean electron density is $3.02 \times 10^{16} \mathrm{~cm}^{-3}$ under 2 Torr in the ablation chamber.
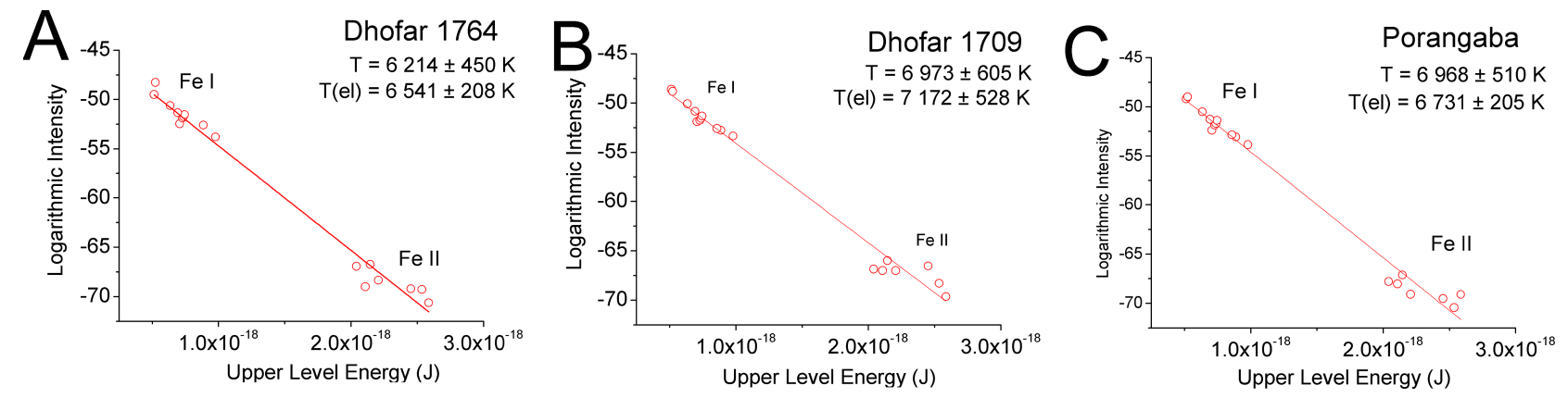

Fig. 3. Saha-Boltzmann plot of selected Fe I and Fe II lines. The values are calculated following substitutions into Eq. (3), see Eqs. (6)-(13).

Using CF data processing according to Eqs. (11)-(14), based on (15) and normalized using (16), we calculated the mixing ratios of the elements (relative to $\mathrm{Mg}$ ) using the most prominent emission lines, specifically $\mathrm{Na}, \mathrm{Mg}, \mathrm{Fe}, \mathrm{Ni}, \mathrm{Cr}, \mathrm{Al}, \mathrm{Si}$, and $\mathrm{Ca}$. All the results are shown in Table 1, as well as the results of further analysis of the meteor spectra.

\subsection{Comparative qualitative measurements of LIBS and glow discharge in air}

The spectra of laser-induced breakdown and discharges in air are measured and assigned to compare experimental data with the atmospheric airglow observed in actual meteor spectra. In the discharge, we detected a strong emission spectrum of $\mathrm{N}_{2}$ $\left(\mathrm{C}^{3} \Pi_{u}-\mathrm{B}^{3} \Pi_{q}\right.$, approximately $330 \mathrm{~nm}$, the second positive band; $\mathrm{B}^{3} \Pi_{g}-\mathrm{A}^{3} \Pi_{u}^{+}$, approximately $650 \mathrm{~nm}$, the first positive band) and the very weak emission of $\mathrm{N}_{2}^{+}\left(\mathrm{B}^{2} \Sigma_{u}^{+}-\mathrm{X}^{2} \Sigma_{g}^{+}\right.$, first negative band) (Mangina et al. 2011). In the LIDB, the most prominent spectral features belong to the atomic species such as ions $\mathrm{O}$ II, and N II, together with neutral $\mathrm{N}$ I and $\mathrm{O}$ I above $700 \mathrm{~nm}$ (Camacho et al. 2007). The spectra are shown in Fig. 4. We would like to note that the LIDB airglow emission is from the same species that produce auroral emission. The spectrum, including the transitions shown, is very similar to the spectra shown in the series of papers by Gattinger et al. (2010), although the relative intensities are different. This would suggest that the methods involved with LIDB could also be useful for investigating the auroral plasmas and their emission mechanisms. The line positions were used for the further qualitative spectral assignment of meteor spectra.

\subsection{Analysis of Perseid and Leonid bright bolides}

We have demonstrated above the simultaneous measurement of the LIBS spectra of chondritic meteorites using a high-resolution echelle spectrograph and meteor spectral camera, as well as subsequent $\mathrm{CF}$ calculation of their elemental composition. Images of Leonid and Perseid spectral lines measured using the meteor spectral camera, along with the spectra of the meteorite specimens' ablation, are shown in Fig. 5. Similar to LIBS, we can observe clearly visible lines of Ca I, Mg I, Fe I, and Na I. Additionally, meteor spectra exhibit the strong emission of ionized species Ca II, Mg II, and Si II, together with atmospheric N I, and O I. We can see that although the ionized species of Ca II, Mg II, and Si II can be detected using LIBS as well, they are not as dominant as those in the meteor spectrum.

This qualitative difference can be caused by the hightemperature second component of the meteor spectrum. This effect was discovered and described by Borovička (Borovička 1993, 1994; Berezhnoy \& Borovicka 2010). The main component 
Table 1. Results of CF meteor plasma composition analysis in comparison with the selected results of other authors.

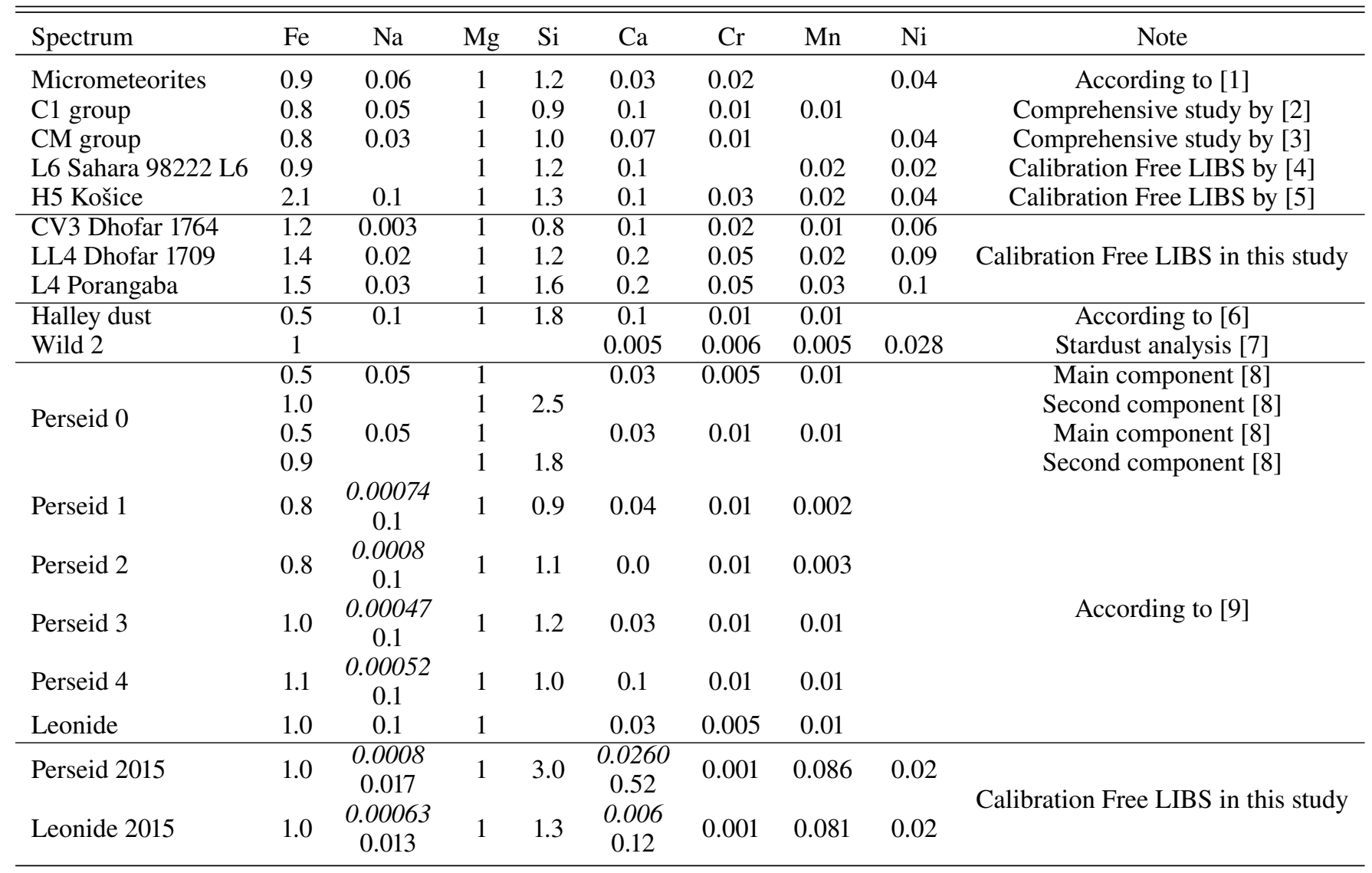

Notes. Uncorrected and corrected data for $\mathrm{Na}$ and $\mathrm{Ca}$ are marked in italics and standard font, respectively. The estimated uncertainty of our fit is $21 \%$.

References. [1] Schramm et al. (1989); [2] Anders \& Grevesse (1989); [3] Mason (1967); [4] Dell'Aglio et al. (2014); [5] Ozdin et al. (2015); [6] Jessberger et al. (1988); [7] Flynn et al. (2006); [8] Borovička \& Betlem (1997); [9] Trigo-Rodriguez et al. (2003).

exhibits an excitation temperature of about $4500 \mathrm{~K}$. However, even higher excitation temperatures of $10000 \mathrm{~K}$ can be estimated for the second component, which is probably connected with the shock wave prominent in fast and bright meteors (as the members of Perseid or Leonid meteor showers). Typical lines of the second component are precisely high excitation lines of $\mathrm{H} \mathrm{I}, \mathrm{Mg}$ II, $\mathrm{Si}$ II, or Ca II. The dominant emission of neutral atoms from the meteor plasma comes from the main component. This emission is partly covered by the second high temperature component, which makes up only 0.02 of the meteor vapour envelope in slow meteors but accounts for more than 5 in fast meteors (Borovička 1994).

Further analysis of the meteor spectra was performed after the conversion of the photographic record to a standard spectrum. Both spectra are shown by the black thick lines in Fig. 6 , panels $\mathrm{A}$ and $\mathrm{B}$. Using our LIBS experimental results, we assigned qualitatively all the spectral features. If we focus on comparative spectra of the meteorite LIBS analysis, discharges and LIDB in the air shown in Fig. 6, panels $\mathrm{C}\left(\mathrm{N}_{2}\right.$ bands in the air discharge) and $\mathrm{D}$, we can observe a roughly similar shape of the main spectral features of $\mathrm{Cr}, \mathrm{Fe}, \mathrm{Ca}, \mathrm{Mg}$ with laboratory data, $\mathrm{Na}, \mathrm{Si}$, and atmospheric volatiles $\mathrm{N}$ and $\mathrm{O}$, together with a strong $\mathrm{H}$ Balmer $\alpha$ line. However, after detailed observation, several lines are determined to differ in intensity. This difference is caused mainly by the diverse temperatures of the meteor and ablation plasma. In fact, the influence of temperature on the shape of emission spectra is crucial. Its value does not only affect the exponential part in Eq. (1) but also plays a role in the calculation of the partition function. The results shown in Fig. 6 are in agreement with the current literature (Borovička \& Betlem 1997; Borovička et al. 1999; Carbary et al. 2003; Trigo-Rodriguez et al. 2003, 2004; Jenniskens 2007; Madiedo et al. 2014a,b; Vojacek et al. 2015).

During further $\mathrm{CF}$ analysis, a calculation similar to that used to determine the composition of Dhofar 1764, Dhofar 1709 and Porangaba meteorite specimen was applied (see Table 1). We fitted the positions and intensities of the most prominent lines (plotted in the red stick diagram for both bolides - Leonid and Perseid, see Fig. 6) of MgI, Si I, Fe I, Na I, CaI, CrI, MnI, and $\mathrm{Ni}$ I and plotted them in a Boltzmann plane. The result of the fit is shown Fig. 7, panels A and B. In the Fig. 7, the red stick diagram gives the position and intensity of 417 lines fitted using the experimental data; the red envelope corresponds to the result of the entire convolution at the given line parameters and experimental correction corresponding to the ablation and meteor spectral data. We note that experimental positions and intensities of these 417 lines were recorded simultaneously by a high-resolution echelle spectrograph, after which the convolution of their intensities was calculated using a programme called Fytik, while at the same time being calibrated to the meteor spectral camera observations. This dataset was used to fit both the temperature and atomic mixing ratios for the meteor spectrum, 


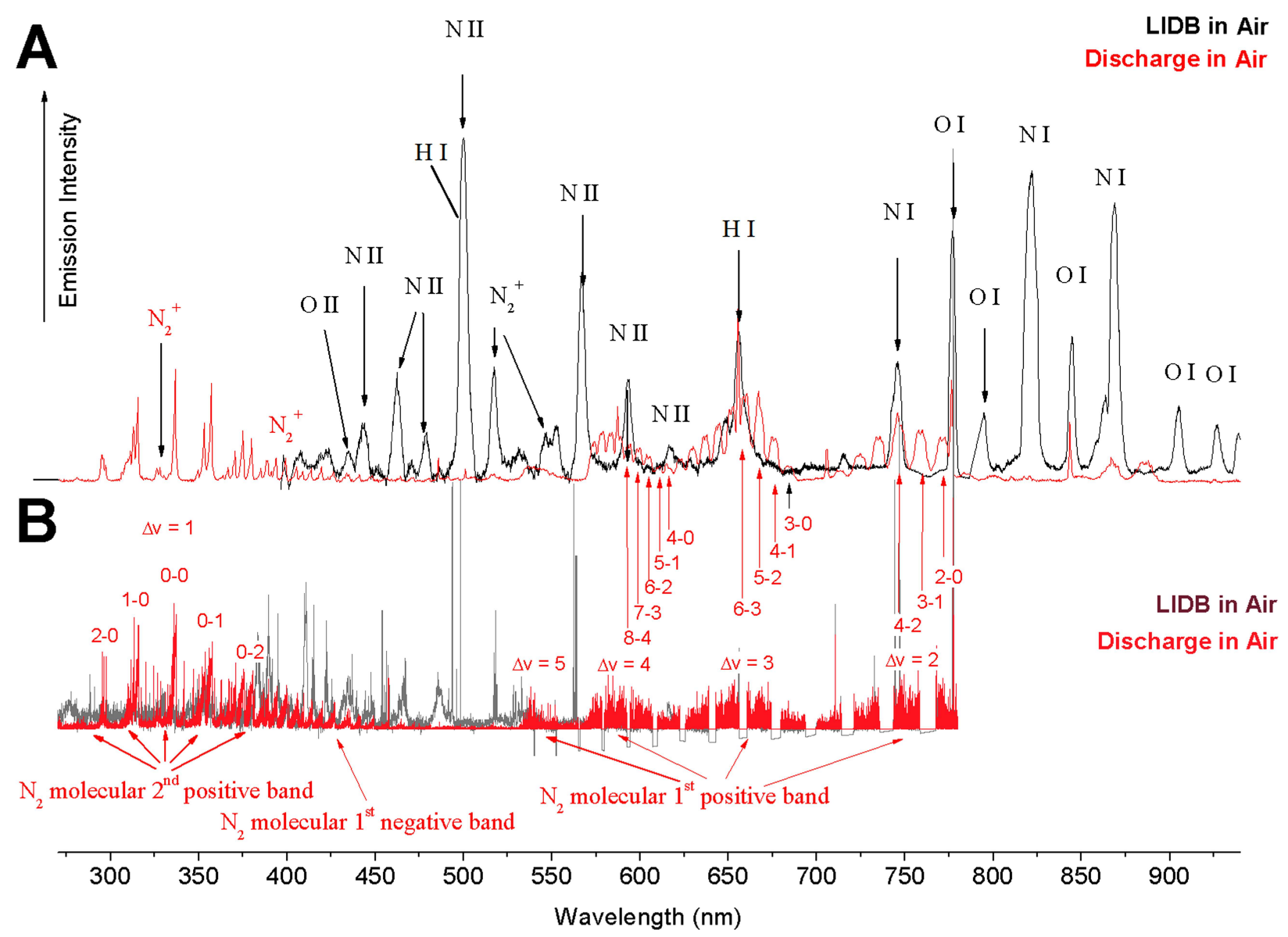

Fig. 4. Panel A: low-resolution spectrum recorded using a meteor spectral camera; panel $B$ : high-resolution spectrum measured using the echelle high-resolution spectrograph. Spectra shown in black and grey belong to laser-induced breakdown in the air; those in red belong to electric discharge. The most prominent atomic species (black letters in panel $A)$ and molecular bands $\left(\mathrm{N}_{2}\right.$ and $\left.\mathrm{N}_{2}^{+}\right)$are assigned in panels $A$ and $B$, respectively.

also using Fytik. Based on these fits, we reconstructed the Boltzmann plane according to the CF Eq. (14), where the values on the $Y$-axes correspond to the calculated logarithmic intensity of an individual line in our fit, and values on the $X$-axes correspond to the upper-level energy of the same individual line. The final elemental abundance is again calculated using Eqs. (15) and (16). Figure 7 shows the result of the Boltzmann plane fitting of 417 lines of each individual element. The estimated $R^{2}$ of the Fe fit is 0.85 . However, we should note that elements such as $\mathrm{Cr}, \mathrm{Ni}$, or Mn contribute less to the uncertainty, because they contribute less to the fit itself. On the other hand, for example, the fit to the sodium doublet is realized only by using two lines (the intensities of the others are negligible, which is visible, for example, in the synthetic spectra). We should note that the logarithmic Boltzmann diagram is extremely sensitive to the temperature, such that slight changes in the temperature contribute exponentially greater changes to the parameters used in Eq. (14) for the fit. This is in fact the major limitation of the low resolution measurement of meteor spectra for which convolution of many lines is required.

In our fit, the slope in the Boltzmann plane corresponds to the temperature of $4177 \mathrm{~K}$ for Leonid and $4667 \mathrm{~K}$ for Perseid meteors. The results of our CF analysis are shown in Table 1. Direct calculation of the elemental abundances using this method is in a close agreement with the previously published data (Borovička \& Betlem 1997; Trigo-Rodriguez et al. 2004). Additionally, in complete accordance with their previously published results, our raw data show a discrepancy between the expected and calculated abundance of the atomic ratio of $\mathrm{Ca}$ I and $\mathrm{Na}$ I to $\mathrm{Mg}$ I. According to the published computations (Borovička \& Betlem 1997), we have corrected our data with respect to the ionization of $\mathrm{Ca}$ and $\mathrm{Na}$ along the meteor trajectory. Our results exhibited a higher content of $\mathrm{Na}$ (however, compared to previous studies, still similar in order), but the ratio of $\mathrm{Ca}$ to $\mathrm{Mg}$ is in the exact agreement with the previously published results (Trigo-Rodriguez et al. 2004). Their uncorrected and corrected data, together with the abundances estimated in this study, are shown in italics and bold, respectively, in Table 1.

In Fig. 6 (panel E), we compare a fully synthetic spectrum to our fit of meteor spectra based on a Calibration-Free convolution of experimental line positions and intensities. A synthetic spectrum was calculated using mixing ratios shown in the Table 1 for the Leonide meteor and a temperature of $4177 \mathrm{~K}$. For ions, we have estimated a temperature of $6450 \mathrm{~K}$ for the second component spectra. For these synthetic spectra, we adopted 22000 lines directly from the NIST database (Kramida et al. 2015). In comparison with the fit using only 417 lines detected in the laser ablation, depicted in the panels $\mathrm{A}$ and $\mathrm{B}$, we can see that the agreement between synthetic spectra and meteor spectra is reduced. However, the results are consistent with other works, showing that more complete fit of the spectra in the meteor and plasma ablation spectra is not yet possible due to significant influences of the matrix, plasma opacity, self-absorption and other factors. 


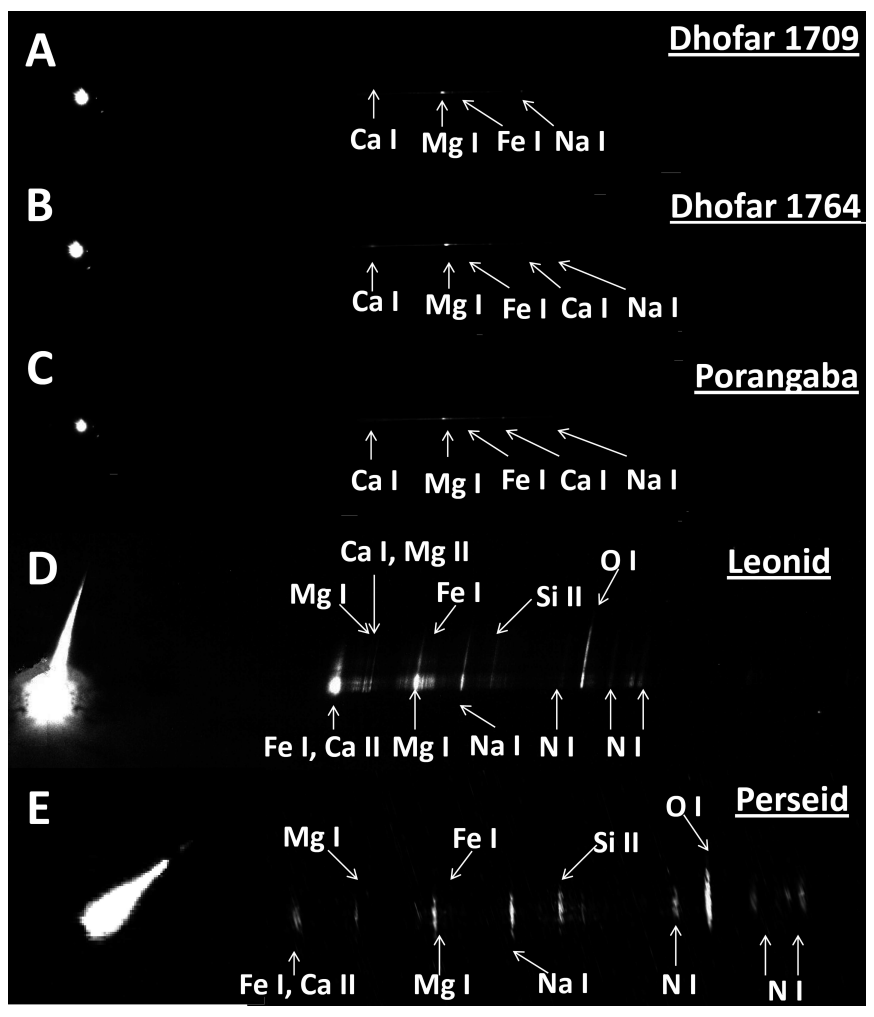

Fig. 5. Panels $A, B$, and $C$ : spectra of laser ablation recorded by a meteor spectral camera during in situ ablation of three samples of chondritic meteorites. Panel $D$ : spectrum summary image of Leonid bolide 20151119_034504, recorded by the meteor spectral camera at the Observatory Valašské Meziříčí, NW camera. Panel E: spectrum of Perseid bolide 20150812_232102, recorded by the mobile meteor spectral camera at the Partizanske station.

Particularly in the UV range, the major discrepancy between the measured (Fig. 6, panels A and B) and synthetic spectra (Fig. 6, panel E) can be explained by low efficiency of the spectrograph, as indicated by the grey line in Fig. 6, panel E. Also, in contrast to longer wavelengths, the measured intensities in the UV range can be affected by atmospheric absorption and the self-absorption.

Although all these effects have been treated carefully (by application of calibration and correction functions), the uncertainty of the measured intensities in the UV region remains significant. In this the advantage of the CF method is clearly demonstrated: The peaks, whose intensities are affected for any of the mentioned reasons, are identified as outliers (represented by points far from the corresponding pyrometric line shown in the Fig. 7) and can be excluded from the further quantitative analysis.

Despite the fact that a conventional CF-LIBS analysis must satisfy several criteria, for example the existence of a plasma in local thermodynamic equilibrium (LTE plasma), our results demonstrate that this is a suitable method for evaluating the composition of meteor plasmas, expected to be close to the composition of the evaporated body itself (if we integrate the spectra along the meteor trajectory neglecting differential ablation of the material). The LTE plasma should exhibit several properties: it should have balanced dynamics, high temperature and density, the population of the excited species should obey the Boltzmann distribution, the velocity distribution should be Maxwellian, the radiation processes should be negligible, and the plasma should be collision-dominated. The McWhirter criterion of LTE plasma conditions defines a minimal electron density in the order of $10^{16} \mathrm{~cm}^{-3}$. Under such conditions, the thermodynamic temperature consequently must be equal to the electron temperature, according to Eq. (6). In meteor plasma, however, electron densities ranging from $2.2 \times 10^{12} \mathrm{~cm}^{-3}$ (see Jenniskens et al. 2002), and $3 \times 10^{12}$ to $2 \times 10^{13} \mathrm{~cm}^{-3}$ (Perseids, (see Borovička \& Betlem 1997), up to $5 \times 10^{14} \mathrm{~cm}^{-3}$ (simulation published by Dyrud et al. (2004)), are expected. In our particular case, the Sudden Ionospheric Disturbance (SID) monitoring station in Carlsbad recorded a peak electron density of $4 \times 10^{14} \mathrm{~cm}^{-3}$ for the Perseid 2015 meteor analysed in this study. However, under such conditions are orders of magnitude below electron densities of of $10^{16} \mathrm{~cm}^{-3}$. However, the explorations performed by Jenniskens (2003) exhibit good agreement with the LTE conditions based on the order-of-magnitude dependency of temperature determined by nitrogen and oxygen emission compared with air plasma models. In the meteor plasma directly around the descending body, such conditions are not necessarily expected due, among other things, to the mainly collisional excitation of compounds. The initial impact excitation is actually a highly non-LTE process.

Ablation starts at elevations ranging from 70 to $110 \mathrm{~km}$ (Baggaley 1980). At these elevations, the mixing ratio of $\mathrm{N}_{2}, \mathrm{O}_{2}$, and $\mathrm{CO}_{2}$ is similar to ambient conditions. The large initial kinetic energy of a descending body is transferred to particles in expanding vapours. Initially, these particles are moving with velocities often much greater than the equivalent width of the Maxwellian velocity distribution of a $4500 \mathrm{~K}$ gas. In fact, for example, Fe atoms should exhibit an average velocity of $1 \mathrm{~km} \mathrm{~s}^{-1}$. However, in case of meteors, we estimated $71.7 \pm 0.3 \mathrm{~km} \mathrm{~s}^{-1}$ for the Leonid meteor and $58.6 \pm 0.3 \mathrm{~km} \mathrm{~s}^{-1}$ for the Perseid meteor. Collisions at this velocity with the atmosphere lead to excitation and ionization, for example in case of $\mathrm{Ca}$

$\mathrm{Ca}+\mathrm{O}_{2}=\mathrm{Ca}^{*}+\mathrm{O}_{2}$,

$\mathrm{Ca}+\mathrm{O}_{2}=\mathrm{Ca}^{+}+\mathrm{e}^{-}+\mathrm{O}_{2}$.

Such processes dominate over dissociative excitation, dissociative ionization and the excitation of atmospheric molecules (Baggaley 1980). It is estimated that excitation (Eq. (22)) dominates over ionization (Eq. (23)). For example, for meteoroid with velocity of $40 \mathrm{~km} \mathrm{~s}^{-1}$, the ratio between atoms and ions was found to be about 100:3 (Sida 1969) (Table 1). Large translational energy of atoms degraded into a hot expanding plasma, cooled down primarily through the energy transfer within collisions of atoms with air molecules. The subsequent electron energy distribution is determined by complex collision processes with electron-electron Coulomb collisions producing a Maxwellian distribution (Baggaley 1980). This thermalization of the system and radiation transfer from those species gets close to the local equilibrium, allowing the determination of mixing ratios using spectroscopic methods (Jenniskens et al. 2004; Jenniskens \& Stenbaek-Nielsen 2004). This process is different from LIBS, because in laser plasma, atoms are vaporized from the surface being heated up to about $5000 \mathrm{~K}$ by laser radiation and then they absorb this radiation in the gas phase, creating breakdown. Excitation is realized by multiphoton ionization, producing electrons. In such a case, LTE is achieved at a sufficient electron density. The distribution of excitations by electrons is equilibrated in the plasma over a time scale of milliseconds, followed by continuum Bremsstrahlung, after the laser pulse with duration in order of nanoseconds. The system is thermalized by collisions. In a laser 


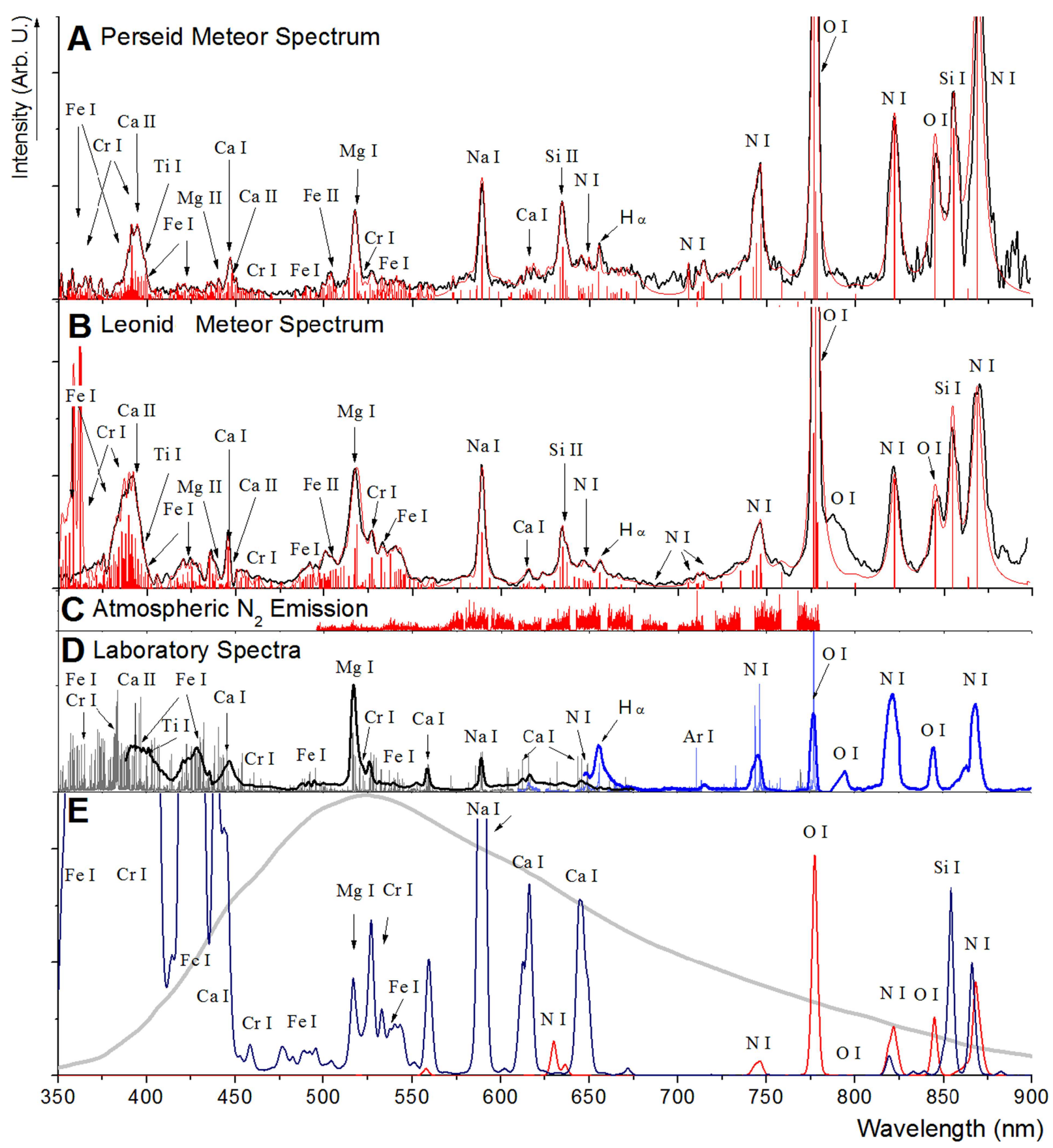

Fig. 6. Panels $A$ and $B$ : assignment of the Perseid and Leonid bolides spectra (marked in black), together with the convolution of lines (intensity and position displayed in stick diagram), simulated using the Fytik programme and a preliminary version of Meteor Master. For clarity, panel $C$ shows separately the positions and intensities of the molecular nitrogen spectrum used in the convolution model. Panel D: experimental spectrum of the laser ablation of chondrite, calculated as a sum of all the samples measured in this study. The high-resolution spectrum, recorded using an echelle spectrograph, is marked in grey; the low-resolution experimental ablation spectrum, recorded in situ by a meteor camera, is shown in black. Similarly, the LIDB spectrum in the air from the high-resolution echelle is depicted in blue, while the low-resolution spectrum from the camera is depicted in dark blue. Panel E: synthetic spectra of the Leonide meteor computed using approximately 22000 lines from the NIST database (Kramida et al. 2015) for temperature of $4667 \mathrm{~K}$ and electron density of $4 \times 10^{14} \mathrm{~cm}^{-3}$. The blue line represents the simulated spectra of the Leonide meteor (abundances according to Table 1), the red line shows airglow of $78 \% \mathrm{~N}$ and $21 \% \mathrm{O}$ and the grey line illustrates the quantum efficiency of the meteor spectral camera. Intensities of Ca I, Fe I and Cr I above $450 \mathrm{~nm}$ are artificially suppressed by the instrument.

plasma, electrons are energy mediators created by multiphoton ionization processes. In spite of the fact that LTE laser plasma is generated by different way than meteor plasma, both systems exhibit LTE under specific conditions and CF LIBS analysis of real meteorite samples show that this data evaluation method, together with the experimental method itself, can facilitate the determination of the chemical composition of a meteor plasma.
In agreement with the previous results (Borovička \& Betlem 1997; Borovička et al. 1999; Trigo-Rodriguez et al. 2003, 2004; Jenniskens 2007), we also see a relation between the composition of chondrites analysed in this study using the CF-LIBS analysis and the composition of the meteor plasma calculated using the method presented here. The mean elemental ratio among refractory elements, such as $\mathrm{Mg}, \mathrm{Si}, \mathrm{Cr}, \mathrm{Fe}$, and $\mathrm{Ca}$, suggests 

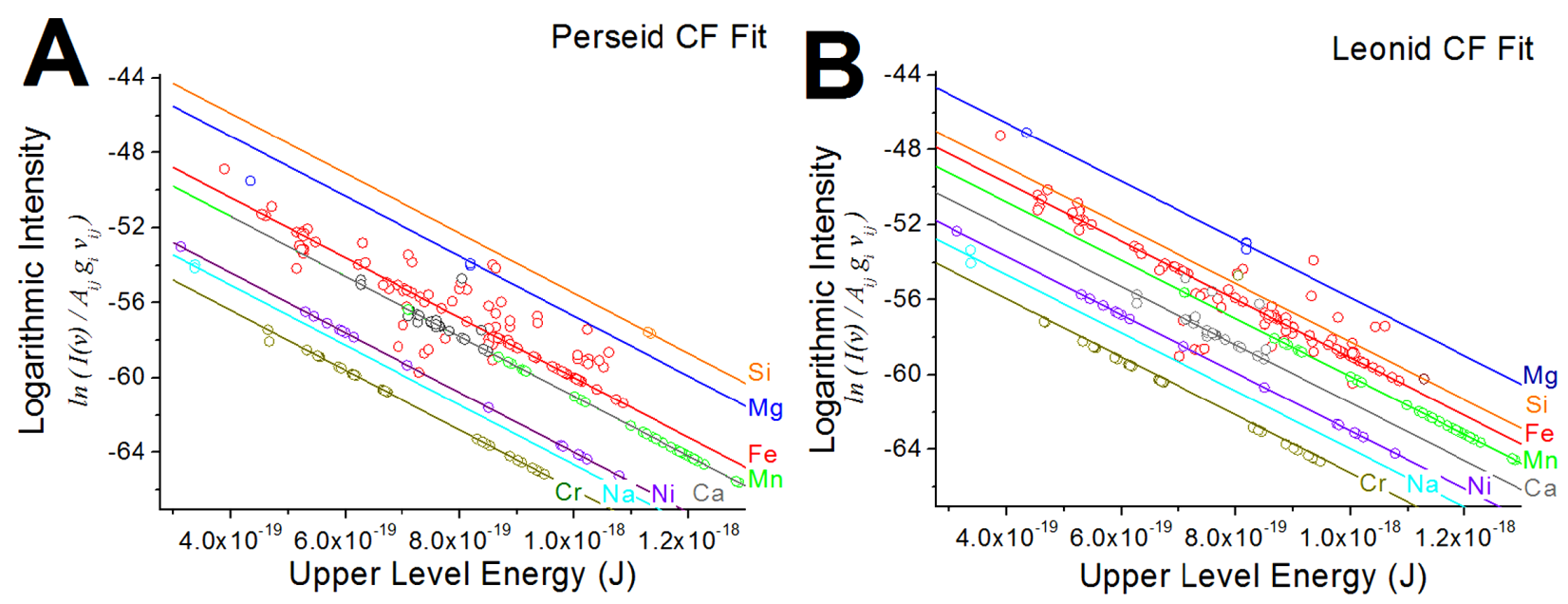

Fig. 7. Graphical results of the CF analysis calculation of Perseid (panel A) and Leonid (panel B) meteor elemental abundances. Each line represents the calculation of the Boltzmann plane. Its slope is (5) reciprocally equal to the temperature; while the intercept represents the $q_{s}$ factor, which is proportional to the elemental abundances.

a chondritic composition consistent with bulk primitive solar system material (e.g. Brownlee et al. 2012) (see Table 1). The only discrepancy is visible in the Leonid Mn-content and Perseid $\mathrm{Si}$-content. In addition to these findings, it is clear that the direct extrapolation of the composition of these small-scale dust particles to larger solids is hardly possible. The low-abundance compounds can be found in different and varying mixing ratios. This is due to the hierarchical structure of the larger particles, which can contain many small pieces of different composition and history. Moreover, the differential ablation may also play a role - the most volatile constituents should start evaporating earlier (i.e. at lower temperature). For example, slower and smaller meteoroids may show enhanced $\mathrm{Na}$ lines in comparison with other more refractory elements (Borovička et al. 2007; Vondrak et al. 2008). In general, taking this fact into account, variation in the exact results of spectral chemical analysis of particular meteors should be expected.

\section{Conclusion}

We compare measurements of the laser ablation spectra of meteorite samples (LIBS), laser-induced breakdown plasma in the air (LIDB) and electric discharge (ED) in the air, and show that similar spectral features can be qualitatively observed in the spectra of Leonid and Perseid meteors. Moreover, we have employed the Calibration Free method for the calculation of the elemental composition using the LIBS spectra for the analysis of Perseid and Leonid meteors. Both spectra are plotted in one Boltzmann plane, and the elemental composition is calculated from individual $q_{s}$ factors. The excitation temperatures were calculated using the bright $\mathrm{Fe}$ I spectral lines and resulted in $4177 \mathrm{~K}$ for Leonid and $4667 \mathrm{~K}$ for Perseid meteors, respectively. The data are compared with the CF analysis of meteorite LIBS spectra, recorded for comparison in our laboratory, and previous results estimated by other authors (see Table 1). The results give good agreement with the previous analyses. Additionally, the compositions of Perseid and Leonid meteoroids are comparable, given that the ratio of $\mathrm{Fe}$ to $\mathrm{Mg}$ is approximately 1, corresponding to the $\mathrm{C}$-group of chondrites. Moreover, the ratio among other elements is very close to the values found for chondritic material. We also discovered, in agreement with other authors, that the predicted ratio of $\mathrm{Na}$ and $\mathrm{Ca}$ to $\mathrm{Mg}$ is influenced by the ionization of these elements within the meteor plasma but is still of the same order of $10^{-1}$ to $10^{-2}$ found in comparative analysis. The $\mathrm{Mn}$ and $\mathrm{Ni}$ to $\mathrm{Mg}$ ratio is found to be of the order of $10^{-2}$ in agreement to previous analyses. However, the Leonid spectrum is poor in $\mathrm{Mn}$ and $\mathrm{Ni}$ and the mixing ratio of $\mathrm{Si}$ is comparable with that of other spectra. On the other hand, we find that the Perseid spectrum is rich in Si compared with other measurements (see Table 1).

Our results show that experiments using the laser-induced ablation of meteorite specimens conducted under strictly defined laboratory conditions can be used for the qualitative assignment of meteor spectra. Within such experiments, elements are evaporated together with the whole matrix, like during the meteoroid descent, and recorded high-resolution emission spectra contain the main lines featured also in the meteor spectra. In our study, we subsequently convolved the high-resolution lines and corrected their intensities using a comparison with the in situ measurement of the same LIBS spectra by a spectral camera for observations of meteors. Using CF analysis, a set of the resulting 417 most significant lines has been used to fit both the temperature and atomic mixing ratios in meteor spectra. The results are in agreement with previously published analysis performed using synthetic spectra and correspond to the compositions expected for C-group chondrites. In our opinion, the results of such an analysis using experimental data, instead of a fully synthetic fit, can significantly contribute to the understanding of meteor spectra and elemental analysis of their plasma, if the experimental LIBS is measured for a sample of meteorite alongside the spectrum recorded during its atmospheric descent. In such a case, reference measurements using other analytical methods (EDX microscopy) can be performed in order to confirm the results of laboratory LIBS, which can be subsequently used to fit the meteor spectrum. Unfortunately, such data are available only for two cases worldwide: Žd'ár nad Sázavou (9th of December, 2014), and Neuschwanstein (4th of April, 2002) meteors (Ferus 2015, priv. comm. with Ing. Lenža and Dr. Borovička).

The importance of laboratory studies such as the ones mentioned in this paper can be demonstrated if we move to other wavelengths. Besides of the optical region most relevant for this current study, additional high resolution comparative laboratory measurements in the ultraviolet would be an essential 
complement to future observations by, for example, the proposed METOR CubeSat satellite (Ortega Varela De Seijas 2017). This device, now in early planning, would observe meteors from Earths' orbit within the UV spectral range - a regime where the emitted light is significantly suppressed due to atmospheric absorption and scattering, as indicated in the Fig. 6. Conversely, in regions of longer wavelengths, infrared Rydberg transitions in atomic spectra can be also observed by satellites. At infrared wavelengths, we can identify the spectral signatures of those radicals and ions produced by the decomposition of organic refractory material (Civiš et al. 2012c) which are nearly impossible to observe at UV or VIS wavelengths (Berezhnoy \& Borovicka 2010). The potential for atomic emission at infrared wavelengths is presently under experimental exploration, for example (Civiš et al. 2012a,b,c,d).

Acknowledgements. The research was funded by the Grant Agency of the Czech Republic (reg. no. 14-12010S and partially also by reg. no. 18-27653S) by the Program of Regional Cooperation between the Regions and the Institutes of the Czech Academy of Sciences in 2017, (reg. no. R200401521 and reg. no. R200401721) and by the STS Missions programme within the COST Actions CM1104 and TD1308. Financial support from the Internal Fund of the J. Heyrovský Institute of Physical Chemistry is also greatly acknowledged. PR acknowledges the Simons Foundation and the Kavli Foundation for funding. EZ is grateful for support from the RFBR (research project No. 16-32-00034).

\section{References}

Abe, S., Ebizuka, N., Murayama, H., et al. 2005, Earth Moon Planet, 95, 265 Anders, E., \& Grevesse, N. 1989, Geochim. Cosmochim. Acta, 53, 197

Baggaley, W. J. 1980, in Meteors and Atmospheres. IAU Symp., 90, 85

Berezhnoy, A. A., \& Borovicka, J. 2010, Icarus, 210, 150

Borovička, J. 1993, A\&A, 279, 627

Borovička, J. 1994, Planet. Space Sci., 42, 145

Borovička, J., \& Berezhnoy, A. A. 2016, Icarus, 278, 248

Borovička, J., \& Betlem, H. 1997, Planet. Space Sci., 45, 563

Borovička, J., Stork, R., \& Bocek, J. 1999, Meteor. Planet. Sci., 34, 987

Borovička, J., Spurny, P., \& Koten, P. 2007, A\&A, 473, 661

Brownlee, D., Joswiak, D., \& Matrajt, G. 2012, Meteor. Planet. Sci., 47, 453

Camacho, J. J., Poyato, J. M. L., Diaz, L., \& Santos, M. 2007, J. Phys. B, 40, 4573

Carbary, J., Morrison, D., Romick, G., \& Yee, J. 2003, Icarus, 161, 223

Ciucci, A., Corsi, M., Palleschi, V., et al. 1999, Appl. Spectrosc., 53, 960

Civiš, S., Ferus, M., Kubelik, P., Chernov, V. E., \& Zanozina, E. M. 2012a, A\&A, 545, A61

Civiš, S., Ferus, M., Kubelik, P., Jelinek, P., \& Chernov, V. E. 2012b, A\&A, 541, A125

Civiš, S., Ferus, M., Kubelik, P., et al. 2012c, J. Opt. Soc. Am. B, 29, 1112
Civiš, S., Ferus, M., Kubelik, P., et al. 2012d, A\&A, 542, A35

De Giacomo, A. 2011, Spectrochim. Acta B, 66, 661

Dell'Aglio, M., De Giacomo, A., Gaudiuso, R., et al. 2010, Geochim. Cosmochim. Acta, 74, 7329

Dell'Aglio, M., De Giacomo, A., Gaudiuso, R., De Pascale, O., \& Longo, S. 2014, Spectrochim. Acta B, 101, 68

Dyrud, L., Denney, K., Close, S., et al. 2004, Atmos. Chem. Phys., 4, 817

Effenberger, Jr., A. J., \& Scott, J. R. 2010, Sensors, 10, 4907

Flynn, G. J., Bleuet, P., Borg, J., et al. 2006, Science, 314, 1731

Gattinger, R. L., Jones, A. V., Degenstein, D. A., \& Llewellyn, E. J. 2010, Can. J. Phys., 88, 559

Hornackova, M., Plavcan, J., Rakovsky, J., et al. 2014, Eur. Phys. J. Appl. Phys., 66, 10702

Iida, Y. 1990, Spectrochim. Acta B, 45, 1353

Jenniskens, P. 2003, The Institute of Space and Astronautical Science Report S.P., 15, 189

Jenniskens, P. 2007, Adv. Space Res., 39, 491

Jenniskens, P., \& Stenbaek-Nielsen, H. 2004, Astrobiology, 4, 95

Jenniskens, P., Tedesco, E., Murthy, J., Laux, C. O., \& Price, S. 2002, Meteor Planet. Sci., 37, 1071

Jenniskens, P., Laux, C. O., Wilson, M. A., \& Shaller, E. L. 2004, Astrobiology, 4,81

Jessberger, E. K., Christoforidis, A., \& Kissel, J. 1988, Nature, 332, 691

Konjević, N. 2002, J. Phys. Chem. Ref. Data, 31, 819

Kramida, A., Ralchenko, Y., Reader, J., \& NIST ASD Team. 2015, NIST Atomic Spectra Database (version 5.5.2)

Madiedo, J. M., Trigo-Rodriguez, J. M., Konovalova, N., et al. 2013, MNRAS, 433, 571

Madiedo, J. M., Ortiz, J. L., Trigo-Rodriguez, J. M., et al. 2014a, Icarus, 231, 356

Madiedo, J. M., Ortiz, J. L., Trigo-Rodriguez, J. M., et al. 2014b, Icarus, 233, 27

Mangina, R. S., Ajello, J. M., West, R. A., \& Dziczek, D. 2011, Astrophys. J. Suppl. Ser., 196, 13

Mason, B. 1967, Am. Sci., 55, 429

Ortega Varela De Seijas, M., Vaubaillon, J. 2017, Analyzing Meteor Composition Using UV Spectroscopy, https://oceanoptics.com/ analyzing-meteor-composition-using-uv-spectroscopy/

Ozdin, D., Plavcan, J., Hornackova, M., et al. 2015, Meteor. Planet. Sci., 50, 864

Schramm, L. S., Brownlee, D. E., \& Wheelock, M. M. 1989, Meteoritics, 24, 99

Sida, D. W. 1969, MNRAS, 143, 37

Takahashi, T., Thornton, B., Ohki, K., \& Sakka, T. 2015, Spectrochim. Acta B, 111,8

The Atomic Linelist, 2016, The Atomic Linelist V2.05B19 (University of Kentucky)

Tognoni, E., Cristoforetti, G., Legnaioli, S., \& Palleschi, V. 2010, Spectrochim. Acta B, 65, 1

Trigo-Rodriguez, J. M., Llorca, J., Borovička, J., \& Fabregat, J. 2003, Meteor. Planet. Sci., 38, 1283

Trigo-Rodriguez, J. M., Llorca, J., \& Fabregat, J. 2004, MNRAS, 348, 802

Vojacek, V., Borovička, J., Koten, P., Spurny, P., \& Stork, R. 2015, A\&A, 580, A67

Vondrak, T., Plane, J. M. C., Broadley, S., \& Janches, D. 2008, Atmos. Chem. Phys., 8, 7015 